\title{
Optimale TIN-Therapie noch nicht gefunden
}

— Die Testikuläre Intraepitheliale Neoplasie (TIN) ist die Vorstufe eines Hodentumors. Wird sie nicht behandelt, entwickeln $70 \%$ der Patienten innerhalb von sieben Jahren ein Seminom. Die therapeutischen Möglichkeiten bei einer TIN sind Abwarten und Beobachten (unverantwortlich, da viele Patienten einen Tumor entwickeln), die Entfernung des Hodens (Übertherapie), eine Cisplatin-basierte Chemotherapie sowie eine Radiatio, erklärte Prof. Klaus-Peter Dieckmann, Hamburg.

Dieckmann stellte eine eigene Studie vor, deren Ziel es war, die verschiedenen Alternativen zu vergleichen. In die retrospektive, multizentrische Studie wurden 186 Patienten mit einem Durchschnittsalter von 30 Jahren eingeschlossen, bei denen ein einseitiger Hodentumor sowie eine histologisch gesicherte TIN im zweiten Hoden vorlagen. 76 Patienten erhielten eine Strahlentherapie
(18-20 Gy), 75 eine Chemotherapie aufgrund von Metastasen, 25 eine Kombination beider Verfahren und zehn wurden nur beobachtet.

Im Rahmen der Nachbeobachtung (im Mittel 30 Monate) wurde der Therapieerfolg sowie das mögliche Auftreten eines Hypogonadismus dokumentiert. Von den bestrahlten Patienten wurde bei 21 eine Kontrollbiopsie durchgeführt, die bei allen negativ ausfiel. 49 Patienten ohne Kontrollbiopsie waren ebenfalls tumorfrei, bei zwei Patienten hatte sich trotz Radiatio ein Seminom entwickelt.

Weniger effektiv war die Chemotherapie. Hier gab es je nach angewendetem Therapieregime etwa 30\% Therapieversager, bei denen entweder eine TIN oder ein Hodentumor nachgewiesen werden konnte. Besonders „Carboplatin bringt fast nichts für die Eradikation der TIN" brachte Dieckmann die Ergebnisse auf den Punkt. Bei Patienten mit einer kom- binierten Chemo- und Strahlentherapie gab es einen Therapieversager $(4,2 \%)$ und in der beobachteten Gruppe waren es wie erwartet $60 \%$.

Ein Hypogonadismus trat bei bestrahlten Patienten in $28 \%$ der Fälle auf; nach Chemo- oder Kombinationstherapie waren $15,6 \%$ beziehungsweise $18,2 \%$ davon betroffen. Auf der Suche nach der optimalen Therapie war somit "leider die Realität anders als der Wunsch" so Dieckmann. „Entweder haben wir eine relativ hohe Versagerquote unter der Chemotherapie oder aber sehr viele endokrinologische Schäden nach einer relativ erfolgreichen Therapie" erklärte der Urologe. Die Daten zeigen, dass die Therapie entweder zu ineffektiv oder aber zu toxisch ist. Weitere Untersuchungen müssen also folgen, so Dieckmann abschließend. as

V7 „Tumoren des äußeren Genitale Hodentumoren“

\section{Gezielte Strategien im Kampf gegen resistente Keime}

— Fast 25.000 Patienten sterben in Europa jährlich an Infektionen, die durch multiresistente Erreger (MRE) verursacht werden, betonte Dr. Florian Wagenlehner, Gießen. Auch in Europa nehme die Zahl der MRE immer weiter zu - in einigen Ländern wurde bereits im Jahr 2008 für manche Erreger eine Resistenz von bis zu $50 \%$ festgestellt. Als Grund für die Zunahme der Resistenzen nannte Wagenlehner die empirische Therapie ohne Antibiogramm: Je höher die Resistenzen, desto eher kommt es zum Therapieversagen. Das wiederum führt zur Verwendung weiterer Breitbandantibiotika, die allgemein ein höheres Potenzial haben, resistente Erreger zu selektionieren. Daten verschiedener Untersuchungen belegen einen klaren Zusammenhang zwischen dem Antibiotikaverbrauch und der Zahl der Resistenzen. Das ist vor allem deshalb problematisch, weil momentan keine neuen Antibiotika zur Verfügung stehen, warnte Wagenlehner.

Was das in Sachen Infektionskontrolle bedeutet, stellte Prof. Axel Kramer,
Greifswald vor. Zur Prävention von Infektionen mit MRE sei es notwendig, Aktivitäten interdisziplinär zu bündeln und zu fokussieren. Dazu gehören neben dem Screening die (virtuelle) Isolierung, wenn möglich die Sanierung sowie eine Multibarrierenstrategie (ein ineinandergreifen der Hygienestandards), die Antibiotikarichtlinie und qualitätssichernde Maßnahmen, so Kramer. Dass eine solche Strategie Erfolg bringen kann, zeigt das Beispiel der Niederlande, in denen durch ein gezieltes Vorgehen die MRSAZahl von $25 \%$ auf unter $1 \%$ gesenkt werde konnten.

Prof. Winfried Kern, Freiburg nannte als wichtige Kriterien einer Antibiotikastrategie die systematische Verbesserung der Verschreibungsqualität (Erstellung von Leitlinien und Verbesserung der Leitlinientreue und -effektivität), Reduktion der Antibiotikadichte

Berüchtigter Problemkeim: Multresistenter Staphyloccus aureus sowie die bessere Definition des intelligenten Einsatzes in der Population anhand weiterer Studien. Daneben sollten laut Kern auch die Möglichkeiten zur Dekolonisierung weiter untersucht werden.

F 10 ,Multiresistente Erreger - die neue Geißel der Urologie?"

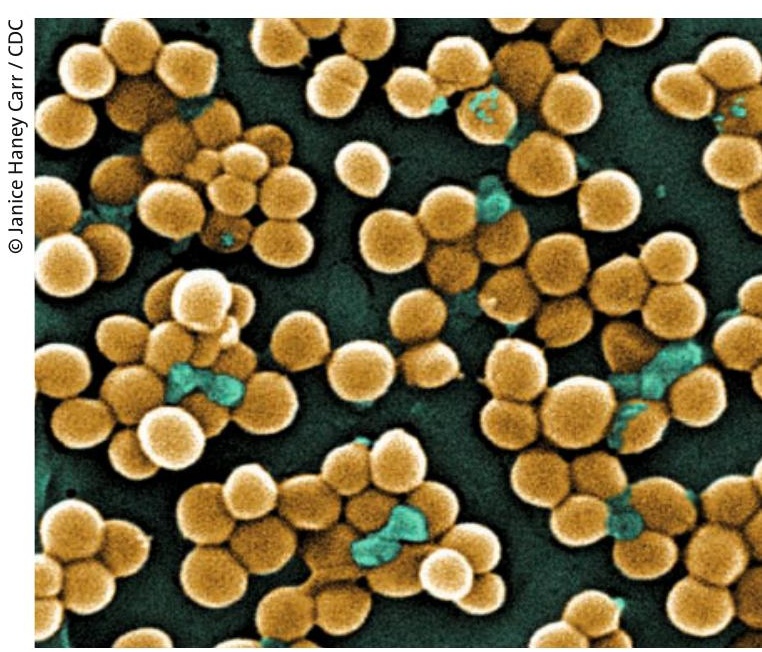

